\title{
Successful Treatment of a Keratoacanthoma with Electrochemotherapy: A Case Report
}

Paola Pasquali · Enrico P. Spugnini · Alfonso Baldi (D)

Received: November 26, 2017 / Published online: January 24, 2018

(c) The Author(s) 2018. This article is an open access publication

\begin{abstract}
Introduction: Few studies have evaluated the efficacy of intralesional bleomycin injection combined with electroporation for the treatment of cutaneous tumors. However, the phenomenon that electroporation can enhance the cytotoxicity of bleomycin in vivo by $300-700$ fold has been intensely investigated.

Case Presentation: Keratoacanthoma in an 86-year-old patient was treated with intralesional bleomycin combined with electroporation. Treatment consisted of local application of shorty and intense electric pulses followed by local injection of bleomycin. Electroporation was always well tolerated by the patient, with
\end{abstract}

Enhanced Content To view enhanced content for this article go to www.medengine.com/Redeem/ EF3B4F6006DB33F8.

P. Pasquali

Dermatology Service, Pius Hospital De Valls,

Tarragona, Spain

E. P. Spugnini

Biopulse Srl, Naples, Italy

A. Baldi $(\square)$

Department of Environmental, Biological and

Pharmaceutical Sciences and Technologies,

University of Campania "Luigi Vanvitelli", Caserta,

Italy

e-mail: alfonsobaldi@tiscali.it no significant complaints, and the tumor had completely regressed by day 71 of the follow-up. Conclusion: The results suggest that intralesional bleomycin injection combined with electroporation could represent a valid alternative therapeutic approach for the treatment of keratoacanthomas.

Keywords: Bleomycin;

Electroporation; Keratoacanthoma; Onkodisruptor

\section{INTRODUCTION}

Keratoacanthoma (KA) is a pruritic and rapidly growing cutaneous neoplasm that appears most frequently in sun-exposed regions and intertriginous areas of elderly people [1]. It bears a close histopathological similarity to squamous cell carcinoma (SCC) and is essentially classified as a low-risk SCC originating from the hair follicle [1]. The predilection for sun-exposed sites supports a pathogenetic role for ultraviolet radiation in KA. The National Comprehensive Cancer Network guideline recommends wide surgical excision or Mohs micrographic surgery for the treatment of local, low-risk SCC [1]. Alternative treatments for patients refusing surgery or for neoplasms arising in areas where surgery is difficult to perform may be required; these include cryosurgery, intralesional application of 5-fluorouracil, methotrexate, or 
interferon $\alpha-2 \mathrm{a}$, radiation therapy, and systemic oral retinoids $[2,3]$.

Electrochemotherapy (ECT) is a loco-regional therapy characterized by the application of permeabilizing electric pulses on tumors after the systemic or intralesional administration of a chemotherapy drug [4]. ECT is presently used as first-line therapy in an adjuvant fashion in veterinary oncology, since it has been proved to improve the efficacy of chemotherapeutic agents and their uptake by the neoplastic cells, especially the anti-cancer chemotherapy drug bleomycin, resulting in enhanced local control of the neoplasia $[5,6]$. In humans, the use of ECT was initially limited to the palliation of cutaneous metastases of melanoma [7], and several phase II trials are ongoing for different tumor types with promising results [8]. In particular, ECT is emerging as a valid therapeutic and palliative treatment for cutaneous and subcutaneous malignancies, including SCC. In comparison to other standard treatments, such as surgery or radiotherapy, ECT has the additional advantage of rapid action on multiple lesions and with repeated sessions in the absence of significant side effects and with no functional impairment [7]. Recently, our research group has proposed a novel protocol for ECT involving the adoption of bursts of rectangular and biphasic pulses with a selectable period of repetition [9]. This protocol has been shown to decrease the morbidity of the treated animals and to improve the clinical outcome. It has been already successfully used in humans for the treatment of large viral warts [10].

\section{CASE REPORT}

We report the case of a 86-year-old man with an eruptive, pruriginous KA for 3 months on the shoulder. The lesion size was $11.20 \times 12.20 \mathrm{~mm}$ (Fig. 1a, c). Clinical and dermoscopic images of the lesions were taken using a Canon EOS Rebel T6i Twin-Flash RL camera (Canfield Scientific Inc., Parsippany, NJ, USA) followed by punch biopsy to confirm histologically the diagnosis of KA (Fig. 1a'). The patient refused surgical treatment; therefore, an alternative approach involving ECT was proposed. The patient was thoroughly informed about the procedure and signed a consent form. The tumor area was anesthetized with a subcutaneous injection (1 cc) of mepivacaine $2 \%$. The patient was then treated with trains of eight biphasic pulses at a voltage of $700 \mathrm{~V} / \mathrm{cm}$ and pulse repetition frequency of $1 \mathrm{~Hz}$, which lasted $50+50 \mu$ s with a $300-\mu \mathrm{s}$ interpulse (total treatment time per train $3.2 \mathrm{~ms}$ ), generated by an electroporator (Onkodisruptor ${ }^{\circledR}$; Biopulse S.r.l., Naples, Italy). The pulses were delivered using caliper electrodes. Immediately after the pulses, bleomycin at a concentration of $1 \mathrm{mg} / \mathrm{cm}^{3}$ was injected into the tumor at a depth of about $1.5 \mathrm{~mm}$ to a final volume of $1 \mathrm{cc}$. The treatment was well tolerated, and the patient did not report any discomfort after the intervention. Local response was assessed after 10 weeks from the procedure, and an objective response was observed. The patient reported only scar tissue within the total resolution of the pre-existing KA (Fig. 1b, d).

All procedures followed were in accordance with the ethical standards of the responsible committee on human experimentation (ethical code of the Università degli Studi della Campania "L. Vanvitelli", approved with D.R. 992/2012) and with the Helsinki Declaration of 1964, as revised in 2013. Informed consent was obtained from the patient for being included in the study.

\section{DISCUSSION}

Surgical excision is the treatment of choice for the majority of cases of KA. However, surgical treatment can result in functional and aesthetic defects, especially when the lesion is large or located in cutaneous areas that are difficult to treat with surgery. In these latter cases, an effective nonsurgical treatment would be more appropriate. We report here an alternative therapeutic approach for KA, namely, the use of ECT combined with intralesional bleomycin injection. The indications for ECT are primitive or relapsing tumors of the skin or subcutaneous tissue of many different histological types [11]. Tumor size strongly influences the response. 

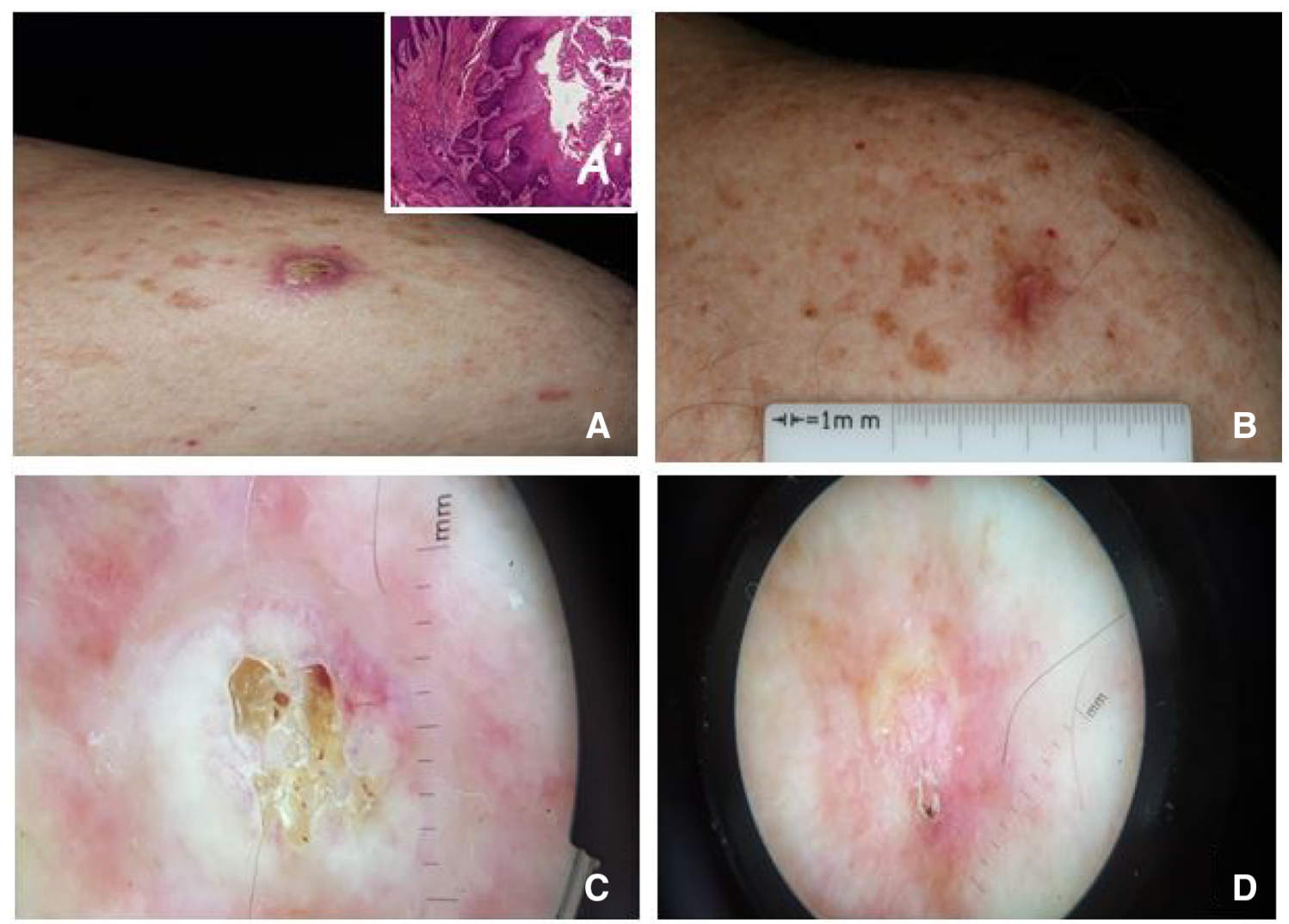

Fig. 1 a Keratoacanthoma at presentation. Inset $\left(\mathbf{a}^{\prime}\right)$ shows the histological appearance of the tumor (hematoxylin/eosin, $\times 10$ ). b Dermoscopy: crateriform central hyperkeratosis. c Appearance at 71-day follow-up

Lesions whose largest diameter does not exceed $3 \mathrm{~cm}$ show a higher complete response rate than neoplasms, as previously described [7]. ECT has been shown in clinical trials to have a high response rate in the treatment of patients with primary or metastatic skin cancers [8]. Interestingly, ECT has been already successfully used to treat eruptive KAs [12]. In the case presented here, the patient showed an excellent response to the ECT treatment and at the moment of writing this report is well. This good result is in accordance with our previous work on ECT treatment of cutaneous pathologies which showed that the majority of patients had no or mild pain after ECT [10]. This study, together with the work of Ribero et al. [12], suggests that ECT can be considered as an excellent alternative to current therapies in the treatment of KA. Nevertheless, it must be considered that KA can

dermoscopy. d Appearance at 71-day follow-up dermoscopy: residual erythema and crusts

spontaneously regress; therefore, more cases are necessary to exactly define the impact of ECT on the clinical history of KA.

\section{CONCLUSIONS}

Electrochemotherapy has several advantages over radiotherapy for the treatment of such conditions as radio-dermatitis, lymphedema, and secondary cancers, including its simplicity and lack of complications [7]. We suggest that it could be an effective alternative treatment for KA, especially for patients who refuse surgery or whose conditions are not suitable for a surgical procedure. Larger, controlled clinical studies are required to confirm its safety and efficacy for the treatment of $\mathrm{KA}$ as well as for other skin malignancies. 


\section{ACKNOWLEDGEMENTS}

Funding. The study and article processing charges were funded by Biopulse Srl.

Authorship. All named authors meet the International Committee of Medical Journal Editors (ICMJE) criteria for authorship for this article, take responsibility for the integrity of the work as a whole, and have given their approval for this version to be published.

Disclosures. EP. Spugnini is a stockholder of Biopulse Srl. A. Baldi is a stock-holder of Biopulse Srl. P. Pasquali has nothing to disclose.

Compliance with Ethics Guidelines. All procedures followed were in accordance with the ethical standards of the responsible committee on human experimentation (ethical code of the Università degli Studi della Campania "L. Vanvitelli", approved with D. R. 992/2012) and with the Helsinki Declaration of 1964, as revised in 2013. Informed consent was obtained from the patient for being included in the study.

Open Access. This article is distributed under the terms of the Creative Commons Attribution-NonCommercial 4.0 International License (http://creativecommons.org/licenses/ by-nc/4.0/), which permits any noncommercial use, distribution, and reproduction in any medium, provided you give appropriate credit to the original author(s) and the source, provide a link to the Creative Commons license, and indicate if changes were made.

\section{REFERENCES}

1. Takai T, Misago N, Murata Y. Natural course of keratoacanthoma and related lesions after partial biopsy: clinical analysis of 66 lesions. J Dermatol. 2015;42:353-62.

2. Yoo MG, Kim IH. Intralesional methotrexate for the treatment of keratoacanthoma: retrospective study and review of the Korean literature. Ann Dermatol. 2014;26:172-6.
3. Pasquali P, Freites-Martinez A, Fortuño-Mar A. Use of cryobiopsy in dermatological practice. J Am Acad Dermatol. 2015;72:e63-4.

4. Spugnini EP, Azzarito T, Fais S, Fanciulli M, Baldi A. Electrochemotherapy as first line cancer treatment: experiences from veterinary medicine in developing novel protocols. Curr Cancer Drug Targets. 2016;16:43-52.

5. Spugnini EP, Baldi A. Electrochemotherapy in veterinary oncology: from rescue to first line therapy. Methods Mol Biol. 2014;1121:247-56.

6. Spugnini EP, Pizzuto M, Filipponi M, Romani L, Vincenzi B, Menicagli F, Lanza A, De Girolamo R, Lomonaco R, Fanciulli M, Spriano G, Baldi A. Electroporatio enhances bleomycin efficacy in cats with periocular carcinoma and advanced squamous cell carcinoma of the head. J Vet Intern Med. 2015;29:1368-75.

7. Sersa G. The state-of-the-art of electrochemotherapy before the ESOPE study: advantages and clinical use. Eur J Cancer Suppl. 2006;4:52-9.

8. Campana LG, Testori A, Curatolo P, Quaglino P, Mocellin S, Framarini M, Borgognoni L, Ascierto PA, Mozzillo N, Guida M, Bucher S, Rotunno R, Marenco F, De Salvo GL, De Paoli A, Rossi CR, Bonadies A. Treatment efficacy with electrochemotherapy: a multi-institutional prospective observational study on 376 patients with superficial tumors. Eur J Surg Oncol. 2016;42:1914-23.

9. Spugnini EP, Melillo A, Quagliuolo L, Boccellino M, Vincenzi B, Pasquali P, Baldi A. Definition of novel electrochemotherapy parameters and validation of their in vitro and in vivo effectiveness. J Cell Physiol. 2014;229:1177-81.

10. Pasquali P, Freites-Martinez A, Gonzalez S, Spugnini EP, Baldi A. Successful treatment of plantar warts with intralesional bleomycin and electroporation: pilot prospective study. Dermatol Pract Concept. 2017;7(3):21-6.

11. Solari N, Spagnolo F, Ponte E, Quaglia A, Lillini R, Battista M, Queirolo P, Cafiero F. Electrochemotherapy for the management of cutaneous and subcutaneous metastasis:a series of 39 patients treated with palliative intent. J Surg Oncol. 2014;109:270-4.

12. Ribero S, Balagna E, Sportoletti Baduel E, Picciotto F, Sanlorenzo M, Fierro MT, Quaglino P, Macripo G. Efficacy of electrochemotherapy for eruptive legs keratoacanthomas. Dermatol Ther. 2016;29:345-8. 